\title{
Design and implementation of operation and maintenance of information systems for Yalong River Hydropower Development Company
}

\author{
Hualong Cai ${ }^{1,2}$, Yuanshou Liu ${ }^{2}$, Yunfeng Liu ${ }^{2}$ \\ ${ }^{1}$ State Key Laboratory of Water Resource and Hydropower Engineering Science, Wuhan University, Wuhan, China \\ ${ }^{2}$ Yalong River Hydropower Development Co., Ltd., Chengdu, China \\ Email address: \\ whucad@whu.edu.cn (Hualong Cai), Liuyuanshou@ylhdc.com.cn (Yuanshou Liu), Liuyunfeng@ylhdc.com.cn (Yunfeng Liu)
}

\section{To cite this article:}

Hualong Cai, Yuanshou Liu, Yunfeng Liu. Design and Implementation of Operation and Maintenance of Information Systems for Yalong River Hydropower Development Company. American Journal of Energy Engineering. Vol. 3, No. 1, 2015, pp. 1-5. doi: 10.11648/j.ajee.20150301.11

\begin{abstract}
Based on the information system of Yalong Company, some analysis are carried out that operation and maintenance of information systems are described covering hardware systems, software systems and the content of individual client behavior management, and analyzes the business logic structure and software architecture system of operation and maintenance, and achieve performance monitoring, security monitoring, supervision and operation and services Desktop system as a whole functions for the overall operation and maintenance information system.
\end{abstract}

Keywords: Yalong River Hydropower Development Co., Ltd., Operation and Maintenance Information System, Design and Implementation

\section{Introduction}

With the putting into production of Ertan, Guandi and the first-second cascade Jinping Hydropower Station and with the construction of Tongzilin, Lianghekou and other power plant, Yalong River Hydropower Development Co., Ltd. (hereinafter referred to as: Yalong Company) gradually comes into a period of rapid development. The information systems of engineering construction, electricity production, operating management as well as other types of business is in use gradually with depth integration of information technology, then the company's information technology operation system has become a dynamic, open, continuous developing and evolving complex system. The operation and maintenance of information technology has very distinctive features with a wide geographic distribution, real-time requirements, diverse content of operation and maintenance. Therefore, the urgent need for information operation and maintenance systems provides an efficient, standardized means of support, and meets some new demands for the mission of systems.

\section{Management Category of Operation and Maintenance Information Systems}

Information system of Yalongcompany includes: financial management systems, human resources management system, OA system, file systems, electrical production management system deployed in a centralized manner; project management system deployed in each construction administration deployed in a distributed manner. Centralized deployment system is released in Chengdu headquarters room, while distributed systems are deployed in each room of construction administration camp. The information operation and maintenance systems covers condition monitoring of hardware equipment such as servers, network, etc.; application monitoring of system software running, monitoring and management on the PC client and the user's personal behavior that is divided into two layers with the centralized deployment and distribution deployment management.

\subsection{Operation and Maintenance Management of Hardware Monitoring}

Operation and maintenance management of hardware monitoring involves: running status monitoring of services, 
status and performance monitoring of network devices and condition monitoring of data flow as shown in Fig.1.

The CPU temperature and usage, memory usage, network cards occupancy rate and other technology parameters are obtained through the program installed on the server, and passed to the background operation and maintenance system that monitors real-timely the value of the switch backplane bandwidth, switching capacity, packet forwarding rate and other performance indicators ; monitors such real-time performance metrics as storage speed storage capacity, CL, SPD chip, parity, memory bandwidth. Set the threshold by the background operation and maintenance management software to provide an early warning mechanism.

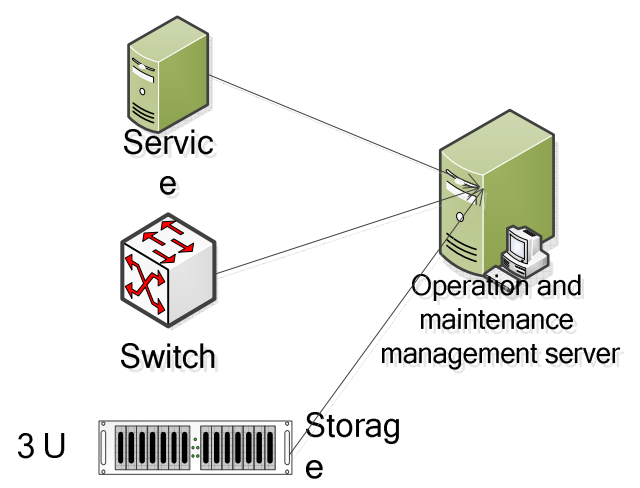

Fig. 1. Real-time hardware monitoring.

\subsection{Operation and Maintenance Management of software Monitoring}

Operation and maintenance management of software monitoring involves: running status monitoring of database, function monitoring of implication layer and condition monitoring of email services, middleware condition monitoring, web service status monitoring, etc. as shown in Fig.2.

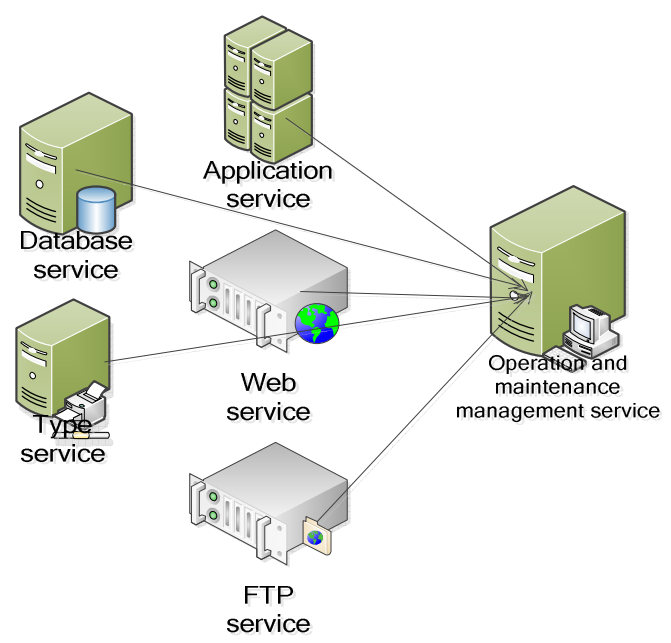

Fig. 2. Real-time software monitoring.

The index such as database response time, throughput performance, Oracle EBS, Maximo, Lotus Domino can be monitored real-timely through software and transmitted to the operation and maintenance systems. By setting the threshold, operation and maintenance systems report to operation and maintenance personnel according to an early warning mechanism.

\subsection{Operation and Maintenance Management of Monitoring User'S Behavior}

Operation and maintenance management of monitoring user's behavior involves: software installation and version control monitoring of PC client, individual user behavior monitoring system and security monitoring of PC client.

For personal computers, laptops, tablet PCs, they are installed with the security software company purchasing, by which some information can be collected example for the software version of terminal device installed, the behavior of individual users of business systems and safety information. The operation and maintenance of information systems analyze and access the indicators of health degree of individual terminals by information collected and send unhealthy terminal information to the mailboxes of the employee using the device and alert maintenance of equipment terminals.

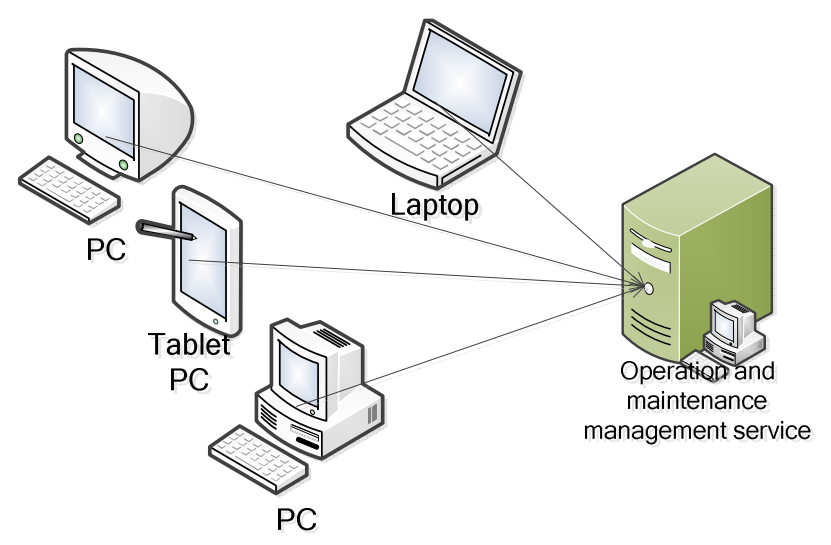

Fig. 3. User's behavior monitoring.

\section{Logical Structure of the System}

The customer service of operation and maintenance information system can be divided into the customer services of ware maintenance, the customer service of equipment maintenance and the desktop customer service. The software customer service is responsible for the operation and maintenance of software applications of trouble ticket resolution; the equipment operation and maintenance customer is responsible for resolving trouble tickets of servers, storage, network and other hardware devices; the desktop customer is responsible for such trouble tickets as system operation and maintenance of the PC client and software configurations. In the presentation layer, in accordance with the contents of the different sub-portals, the customer service can be divided into the operation and maintenance sub-portal, equipment sub-portal, security sub-portal and desktop sub-portal to provide information services for different 
operation and maintenance teams. In the business layer, the configuration, re-optimized work flow can be come true; real-time monitoring of application software, hardware, data acquisition equipment can be applied, desktop information gathering and the comnanv's overall s sm....te infommat gathering can be also achieved along with external systems and data exchange. Business layer achieves operation of the database through the data access layer with storage / operation and maintenance of information, and analyze the results to put $،$ tto 1 - 1_t.... tgain.

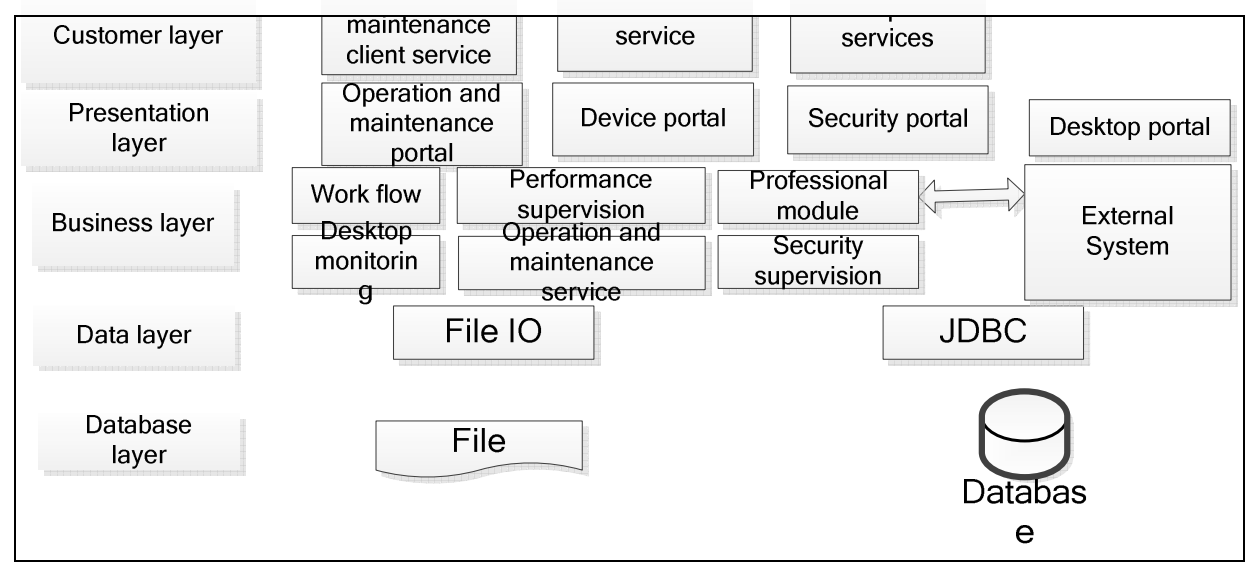

Fig. 4. Logical framework of system.

\section{Software Structure of the System}

Operation and maintenance of information systems is divided into the presentation layer, business layer, database access layer and the database layer. The presentation layer shows the operation and maintenance information through the static and dynamic pages; users interact with the operation and maintenance system via the presentation layer. Business layer achieves communication with presentation layer by Struts MVC Action; reconstruction and implement of work flow comes true through Workflow, business functions can achieve the realization by the Spring framework. Business layer carries out operation of the database by FileIO, JDBC, IBATIS.

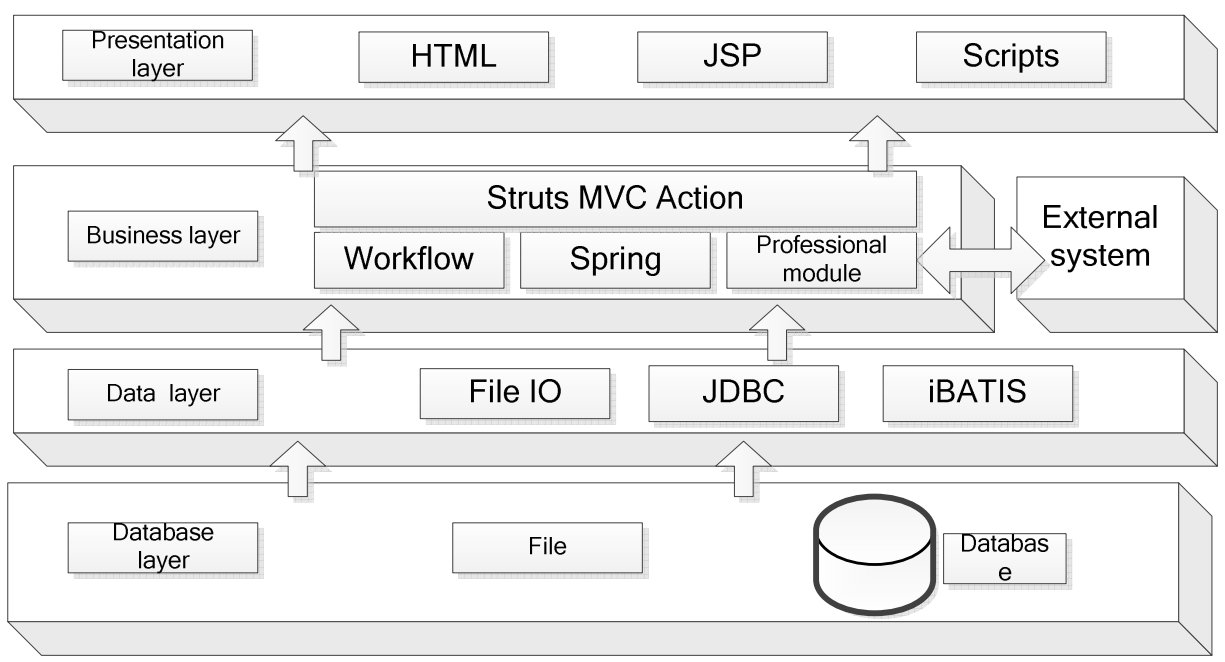

Fig. 5. Software framework of system.

\section{Realization of System Functions}

The functions of information operation and maintenance system of Watershed Company includes performance monitoring, security supervision, Desktop supervision, operation and maintenance supervision,etc.

\subsection{Performance Monitoring}

Performance monitoring refers to the real-time monitoring and management for business systems, middleware, database and network, server rooms and other hardware equipment, facilities. Business regulation includes financial management systems based on Oracle EBS, human resources management system, Maximo-based electricity production management system, file management system based on Unis, Oracle Portal-based portal management system, Lotus Domino based OA system, in which the system real-timely monitors the operation indicators of applications.

Network monitoring can carry out supervision on the network topology, running condition of network switches and other equipment, the port operation of the regulatory company-wide link. The running status of device can be 
monitored and obtained with cross-device type and device manufacturers and the threshold for the health degree of is set as an early warning. Room supervision can get real-time such key indicators as room temperature, humidity, power supply and regulate the host running parameters of the engine room, storage and network devices. Three-dimensional and multi-media technology shows the operational status of the engine room and the ability to achieve an interactive room management.

\subsection{Security Supervision}

Security supervision system provide a security information and status of the entire company network, server, PC client for the operation and maintenance personnel monitor, including the risk warning, failure analysis, strategic release, alarm events, reports, data analysis and reporting capabilities show. By the security software presetting in the server and the client as well as the hardware modules of network equipment, gathering security state information on a company-wide devices, can provide real-time security data and generate early warning reports, so that operation and maintenance personnel can take measures to prevent and handle security incidents.

\subsection{Desktop Supervision}

Desktop supervision includes desktop security management, desktop patch management, software management and desktop assets management. Desktop security management involves the lifecycle terminal-supporting management such as security access, illegal outreach, virus protection, security assessments, user behavior. Software and patch refers to software distribution, configuration management, patch management, alarm and updates. Desktop asset management involves management of software and hardware assets and changes in the status quo.

\subsection{Services of Operation and Maintenance}

\subsubsection{Work Order Management}

Work order is the carrier of operation and maintenance services for the event handling, can be divided into four categories: be accepting work orders, work order processing, archiving pending work orders, work orders . Work order process is as follows:

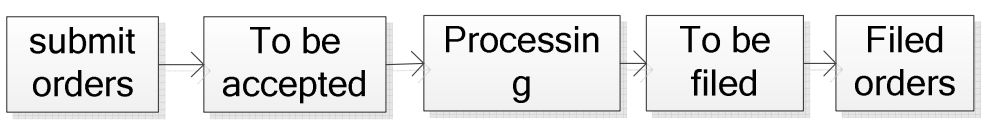

Fig. 6. Process flow of work order.

If users identify problems during running of the system, fill out and submit the issue work orders. The problems in work orders are listed into a single work queue according to the classification, waiting for the operation and maintenance personnel accepted who handle issues raised in each work order. After processing, work orders are waiting for archiving. Confirmed by the records management staff, the work order is for archiving and for future reference. When they find a similar problem, operation and maintenance personnel can call the archived work orders and processing programs reuse.

\subsubsection{Information Distribution}

Operation and maintenance personnel choose the range (the whole company, the Authority, power plants, departments) to publish important notes, spirit of the meeting, personnel and other information of the Authority, power plants, departments in the form of static pages and accessories.

\subsubsection{Operation and Maintenance Assessment}

Classification for the type of work orders and making a statistical analysis for work order processing time, user evaluation and the user visits can make an assessment to operation and maintenance personnel. By the examination, their service quality has been greatly improved. Statistical analysis of data can find the weak link, improve and enhance the overall controlling efficiency of the operation and maintenance system.

\subsubsection{Dissemination of Maintenance Information}

The statistical analysis for operation and maintenance information, the report of operation and maintenance of the quality of service submitted monthly, quarterly, semi-annual, annual are published in the prescribed information bar so that all operation and maintenance personnel can clearly get dynamic operation and maintenance management. Colleagues can also publish information bar operation and maintenance related procedures, norms, easy operation and maintenance personnel can easily find the information you need.Meanwhile, the bar can also publish information related to the operation and maintenance procedures and norms,so operation and maintenance personnel can easily find the information needed.

\subsubsection{Duty Management}

Duty management includes: management on duty arrangements, log management on duty, duty patrol management, room inspection management, shift management, access management, content management, inspection duty. Duty management is able to make a regular basis, qualitative and quantitative operation and maintenance management for the centralized deployment of software and hardware facilities and services of headquarters rooms, data centers, business systems. It is also able to carry out an efficient supervision for related facilities and services of distributed project management system.

\subsubsection{Scheduling Management}

Scheduling management can be divided into the plan preparation, plan generation, plan distributions, implementation of the plan, implementation assessment of the plan. By the plan management module of operation and 
maintenance system, operation and maintenance resources can be configured in the overall company, and gradually formed applications, room management, network management, staffing and other professional jobs plan outline through the accumulation of time, providing a reference for the management of daily operation programming.

\subsubsection{System Management}

The administrators of operation and maintenance system carry out information management of operation and maintenance personnel through the backstage management system, depending on the professional work of each person with different tasks, define different roles within the system, given the appropriate administrative privileges, so that each operation and maintenance resources are able to maximize resource efficiency through operation and maintenance information system.

\section{Conclusion}

Based on running status information systems of the Yalong Company, combined with ITIL framework system, operation and maintenance information systems can achieve business management, service management, infrastructure management, service planning and implementation, application management and security management and so on. The implementation of the operation and maintenance system improves the quality of information systems services to provide an efficient platform support for business entities.

\section{References}

[1] Chen Yuhui. Create a distinctive grid integrated management information system of operation and maintenance $[\mathrm{J}]$, power information,2011(2):165-169

[2] You Zhenwei. Design and implementation of operation and maintenance of electric information systems [J],Jiangxi Communication technology,2011(9): 5-10

[3] Zhao Zhendong. Design and implementation of operation and maintenance of electric information systems [D],Beijing: Beijing University ofposts and telecommunications,2012

[4] Wei Ming. Scheme of ITIL-based information management system of operation and maintenance $[\mathrm{J}]$, power information, 2011(4):83-86.

[5] Li Zhen. Modeling of operation and maintenance of national indicatorsbased on high-dimensional multi-objective optimization of network [J], Computer and Modernization, 2013(6):23-26.

[6] Zhu Ruoming. Design and implementation of monitoring system for internet behavior [D]. Changsha: Central South University,2011 